AeDA/DGAKI Notes

\section{AllergoActiv: Mitmachen erwünscht!}

— Die AllergoActiv-Seminare sorgen mit praktischen Übungen und Anleitungen dafür, dass die kollegiale Interaktion in Bochum nicht zu kurz kommt.

In der täglichen Praxis spielt die Diagnose von allergischen Erkrankungen eine wesentliche Rolle. Im Seminar "Allergiediagnostik auf dem Prüfstand" werden die allergologische Diagnostik im Hautarztverfahren und knifflige Fragen bei der Allergiediagnostik diskutiert. Was tun, wenn z.B. Anamnese, Hauttests und In-vitro-Diagnostik nicht zusammenpassen? Der Schwerpunkt dieser AllergoActiv-Veranstaltung liegt ganz klar im kollegialen Austausch und der Diskussion von echten Kasuistiken und Problemen des täglichen Lebens.

Im dem hoch-aktuellen AllergoActiv "Schimmel - der tägliche Wahnsinn" be-

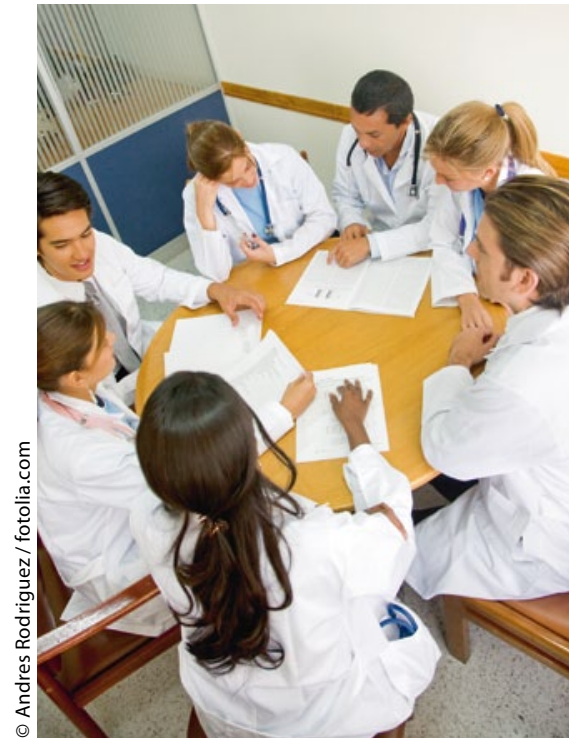

kommen Sie einen Überblick über die unterschiedlichen Erkrankungen, diagnostischen Möglichkeiten sowie Expositionen, die im Zusammenhang mit Schimmelpilzen auftreten können. Durch die Möglichkeit des kollegialen Erfahrungsaustauschs und der Diskussion, die durch das Veranstaltungsformat "AllergoActiv" gewünscht ist, kann diese vielfältige Problematik von unterschiedlichen Seiten beleuchtet werden.

AllergoActiv: Allergiediagnostik auf dem Prüfstand

Freitag, 6. September 2013, 9.00-10.00 Uhr

AllergoActiv: Schimmel der tägliche Wahnsinn

Freitag, 6. September 2013, 10.00-11.00 Uhr

\title{
AllergoCompact: Wichtiges in Kürze
}

— Ein weiteres Veranstaltungsformat des Allergiekongresses in Bochum ist "AllergoCompact". Hier werden die wichtigsten Themen der Allergologie zusammengefasst. So z.B. das Thema Nahrungsmittelallergien. Nahrungsmittelallergien werden immer häufiger und entwickeln sich zu einem zentralen Thema in der Allergologie. Sie stellen für alte und junge Patienten eine wesentliche Beeinträchtigung der täglichen Lebensqualität dar und sind mit dem Risiko von schweren anaphylaktischen Reaktionen behaftet. Ausführlich werden die Fragen der Lebensqualität von Nahrungsmittelallergikern und des Managements von Nahrungsmittelallergie beleuchtet. Daneben stehen kontroverse Themen wie pollenassoziierte Nahrungsmittelallergien oder Histaminunverträglichkeit zur Diskussion.

Beruf und Allergie ist das Thema einer weiteren AllergoCompact-Veranstaltung: Allergene vom Arbeitsplatz und aus der Arbeitswelt können sowohl am Atemtrakt als auch an der Haut zu allergischen Beschwerden führen. Je nach Eintrittspforte und Zielorgan sind es verschiedene Allergene, sodass auch unterschiedliche diagnostische Wege eingeschlagen werden müssen. Der State-of-theart der Diagnostik berufsbedingter Typ-Iund Typ-IV-Allergien wird präsentiert und

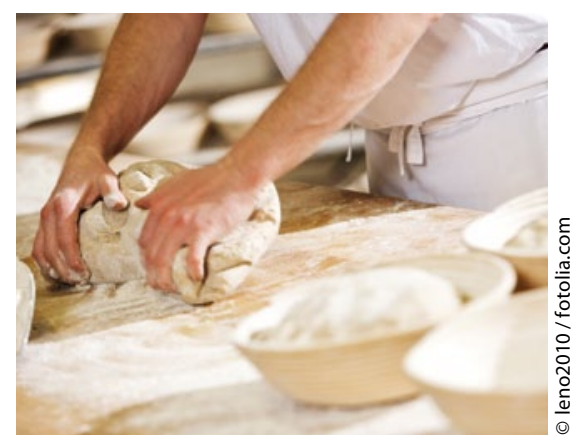

die wichtigsten Werkzeuge für die Prävention allergischer Berufserkrankungen aufgezeigt. Im AllergoCompact „Update - Allergische Rhinitis" wird Ihnen der aktuelle Stand dieser allergischen Erkrankungen in drei interessanten Vorträgen präsentiert. Die Lebens-

qualität der Betroffenen und ihrer Familien, das Konzept der "United Airways" und die neusten Entwicklungen bei Diagnostik und Therapie von nicht-allergischer Rhinitis sind die Themen dieser Veranstaltung.

\section{AllergoCompact: \\ Nahrungsmittelallergie im Focus \\ Donnerstag, 5. September 2013, 9.00-10.30 Uhr \\ AllergoCompact: Update - \\ Allergische Rhinitis \\ Donnerstag, 5. September 2013, 09.00-10.30 Uhr}

AllergoCompact: Beruf und Allergie - Wenn der Beruf allergisch macht

Donnerstag, 5. September 2013, 16.30-18.00 Uhr

\section{Neu! Die E-Poster-Ausstellung}

Die Poster werden in diesem Jahr erstmals im Rahmen einer E-Poster-Ausstellung präsentiert. Neben der eigentlichen digitalen E-Poster-Session werden alle Poster vor, während und nach dem Kongress auf der Kongresshomepage bzw. vor Ort an den E-Poster-Bildschirmen im Großen Saal des RuhrCongress zu sehen sein. Ausgedruckte Poster werden nicht benötigt.

Nähere Informationen zu den technischen Details und dem Upload-Verfahren finden Sie unter www.allergiekongress.de/Abstracteinreichung. 